\title{
Subject Index Vol. 10, 1995
}

Acetylcholinesterase 37 Acidemia 315 Adrenal hyperplasia 311 Alloimmune thrombocytopenia 173 Alloimmunization 373 Ambiguous genitalia 311 Amniocentesis 83, 279, 290, 373 Amniocytes 60 Amnioinfusion 408 Amniorrhexis, preterm prelabor 290 Amniotic fluid 66 Aneuploidy 286 Antenatal ultrasonography 134 Ascites, fetal 200 Asphyxia 1 Associated anomaly 52

Becker muscular dystrophy 71 Betamethasone 301 Bis-diamine 41 Blood gases 141 Bradycardia 315

C-reactive protein 290 Cardiac defects 381 Cerebral flow velocity 106 Chromosomal abnormalities 192, 349

- $\quad$ defects 356

Cobalamin 297

Coelocentesis 66

Coelomic fluid 66

Congenital anomalies 182

cystic adenomatoid malformation, lung 400

diaphragmatic hernia 52,119, 393

Cordocentesis 22, 141, 192,279,

290 -, safety 173 Cost-benefit analysis 157 Creatine kinase isozymes 1 Creating memories 147 Crown-rump length 17 Cytometry 387

Dandy-Walker cyst 333 Delivery 141 Diabetes mellitus 141 Diaphragmatic hernia 307

Discordant twins 17 Doppler 17,111

- $\quad$ ultrasound 315

Down syndrome 131,368

Duchenne muscular dystrophy 71 ,

167 Ductus arteriosus 41

Ebstein's anomaly 22 Electron microscopy 60 Embolization 26 Endoscopy, fetal 167

Epidermolysis bullosa 37 Erythropoietin 141 Estriol 76

Ferritin 297 Fetal anaemia 95

blood sampling 86,326

bradycardia 207

cardiac disease 95

growth restriction 408

- retardation 48

haematology, intra-uterine infection 279

heart rate, sinusoidal pattern, monitoring 207

- - variability 301

hydrops 92,95

hypoxemia 22 
karyotype 101, 349

lung maturation 301

malformations 192

sex 368

surgery 400

testing 157

- $\quad$ therapy 326

Fetal-maternal hydrops syndrome

204

Fetomaternal hemorrhage 48 Fetoplacental passage $7 \alpha$-Fetoprotein 37, 48, 286, 368 Fetoscopy 119 Fetus 41,315,322 Fiberoptic endoscopy 127 Fluorescence in situ hybridisation 66,387

Gastroschisis 119 Genetic amniocentesis 178 Glutathione S-transferase 11 Grief reactions 147

hCG 204 Heart rate 290 Hepatosplenomegaly 131 Human immunodeficiency virus

7 Hybridisation efficiency 66 Hydrops 204

- $\quad$ fetalis 400

Hypertension 111

Hypoxemia 106, 315

Hysterotomy incision 119

Interferon- $\alpha 7$ Intraperitoneal saline infusion

307 Intrauterine fetal death 17

growth retardation 17

stress 1

Intravascular transfusion 173 In utero cystostomy 343

- fetal muscle biopsy 71

- therapy 127 Invasive therapy 101 Isochromosome 387

Karyotype 192 Kleihauer-Betke test 48

Laser surgery 343 Leukocyte count 290 Liver damage 11 Lobectomy 400

417

Macrosomia 134

Marker chromosome aneuploidy,

prenatal diagnosis 182 Maternal age 356,368

,- 35 and over 157

hypothermia 207

temperature 290 Maternal-fetal exchange 322 Mild hydronephrosis 349 Molar pregnancy 337

Molecular analysis 71 Multiple-marker biochemical

screening 286 Muscle biopsy, fetal 167

Needle contact 83

- $\quad$ embryofetoscopy 81

Neonatal surgery 400

Non-immune hydrops 101

Nuchal translucency 381

Nutrition 322

Oligohydramnios 408 Ovarian cyst 200 Oxygen administration 22

Partial triploid mole 337 Parvovirus 204 PentasomyX 333 Percutaneous umbilical cord

catheterization 326 Perinatal Grief Scale 147 Pharmacokinetics 7 Placenta 7, 322

Placental barrier 189 
- $\quad$ intervillous space 322

Platelets 173

Pleural effusions 95 Pleuroamniotic shunt 92 Polymerase chain reaction 373 Pregnancy 7

- $\quad$ sac size 17

Prenatal diagnosis 37, 52, 71, 81, 157,167,192,307,311,333, 337,349, 400

Preterm labor 127

- $\quad$ prelabour amniorrhexis 279

Psychosocial support 147

Pulmonary hypoplasia 119, 400

Pulsatility index 315

Pyelectasia 349

Red blood cell isoimmunisation

297 Retinoids 189 Rhesus disease 373 Right aortic arch 41 Risk counselling 356

Screening 76 Segmentectomy 400 Selective fetal termination 32

feticide 26

termination 186 Serum erythropoietin 86 Simpson-Golabi-Behmel

syndrome 134 Single-needle insertion 178 Small for gestational age 111 Spinabifida 81,83

Spontaneous rupture, membranes

186 Steroid sulfatase deficiency 76 Surfactant 127

Teratogenicity 189 Terminal jeopardy 106 Termination of pregnancy, feto-

pathic reasons 147 Thoracocentesis 92 Thrombocytopenia 373 Torsion 200 Tracheal stenosis 41

Translocation, prenatal diagnosis

387 Trisomy 18381 Twin(s) 86

pregnancy $178,186,337$

transfusion syndrome 32 Twin-to-twin transfusion

syndrome 26,86

Ultrasonography 182,349,381 Ultrasound 192,311,326 Umbilical artery 315 Urethral obstruction

343 Uterine artery 111

Varicella 60

Vascular resistance 315

- $\quad$ ring 41

Visceromegaly 134

$\mathrm{X}$ chromosome $387 \mathrm{X}$-linked ichthyosis 76

418

Subject Index Vol. 10, 1995 